\title{
Mucocutaneous Candidiasis
}

National Cancer Institute

\section{Source}

National Cancer Institute. Mucocutaneous Candidiasis. NCI Thesaurus. Code C35576.

A fungal infection of the skin, nails, oral and vaginal mucosal sites caused by species of the genus Candida. It manifests with white discoloration of the tongue and swelling, redness, and tenderness of the nails. 